This item was submitted to Loughborough's Research Repository by the author.

Items in Figshare are protected by copyright, with all rights reserved, unless otherwise indicated.

\title{
Models for instability in geophysical flows
}

PLEASE CITE THE PUBLISHED VERSION

LICENCE

CC BY-NC-ND 4.0

\section{REPOSITORY RECORD}

Grimshaw, Roger H.J., and Georg A. Gottwald. 2019. "Models for Instability in Geophysical Flows". figshare. https://hdl.handle.net/2134/765. 


\title{
MODELS FOR INSTABILITY IN GEOPHYSICAL FLOWS
}

\author{
Roger Grimshaw \\ Department of Mathematical Sciences \\ Loughborough University, UK \\ R.H.J.Grimshaw@boro.ac.uk \\ Georg Gottwald \\ INLS-CNRS, Sophia-Antipolis, France \\ georg.gottwald@inln.cnrs.fr
}

\begin{abstract}
The generation of instability in inviscid, non-diffusive geophysical flows is generically caused by a resonance between two wave modes. The weakly nonlinear unfolding of this situation is described in the long-wave regime, using a particular two-layer quasi-geostrophic model as an illustrative example. The outcome is a system of two coupled Korteweg-de Vries equations. This system contains a very rich solution set, consisting typically of solitary wave interactions. We will describe some numerical solutions of the coupled Korteweg-de Vries equations, supplemented by perturbation analyses. We also report on some preliminary analogous numerical simulations of the full two-layer quasi-geostrophic system.
\end{abstract}

\section{INTRODUCTION}

In inviscid fluid flows it is well-known that instability generally arises due to a resonance between two waves. Thus, as an appropriate external parameter is varied, the phase speeds of two waves coincide for some critical parameter value. The generic unfolding of this resonance yields either a stable "kissing" configuration, or a "bubble" of instability in the space of the external parameter. There are many examples of this situation (see, for instance, the monograph by Craik (1985) for shear flows, or Baines and Mitsudera (1994) for a discussion of the physical processes involved.

Our concern here is with the unfolding of this resonance in the limit of long waves. Recent work has established that there are two generic 
canonical models (see, for instance, Grimshaw (2000)). For the case when the wave modes coincide at criticality, the canonical model is the Boussinesq equation

$$
A_{t t}-\Delta^{2} A_{x x}+\frac{1}{2} \mu\left(A^{2}\right)_{x x}+\lambda A_{x x x x}=\delta A_{x x},
$$

Here $\Delta$ is the aforementioned external parameter, $\delta$ is an unfolding parameter, while $\mu$ and $\lambda$ are the nonlinear and dispersive coefficients respectively. In the linear, long-wave limit the dispersion relation for waves of speed $c$ is just $c^{2}=\Delta^{2}+\delta$. Resonance occurs for $\Delta=\delta=0$, and the flow is linearly stable, or unstable, according as $\delta>0$, or $<0$. Equations of this form have been derived by Hickernell (1938a, b) for Kelvin-Helmholtz instability, and by Helfrich and Pedlosky (1993) and Mitsudera (1994) for certain quasi-geostrophic flows.

However, our main interest here is with the alternative scenario, when the wave modes remain distinct at criticality. In this case a suitable canonical model consists of the coupled Korteweg-de-Vries (KdV) equations

$$
\begin{aligned}
& A_{t}+\Delta_{1} A_{x}+\mu_{1} A A_{x}+\lambda_{1} A_{x x x}+\kappa_{1} B_{x}=0, \\
& B_{t}+\Delta_{2} B_{x}+\mu_{2} B B_{x}+\lambda_{2} B_{x x x}+\kappa_{2} A_{x}=0,
\end{aligned}
$$

Here $\Delta_{1}-\Delta_{2}$ is the detuning parameter, $\kappa_{1}, \kappa_{2}$ are unfolding parameters, while $\mu_{1}, \mu_{2}$ and $\lambda_{1}, \lambda_{2}$ are nonlinear and dispersive coefficients respectively. In the linear long-wave limit the dispersion relation for waves of speed $c$ is

$$
\left(c-\Delta_{1}\right)\left(c-\Delta_{2}\right)=\kappa_{1} \kappa_{2} .
$$

This is equivalent to that for the Boussinesq equation (1.1) if we put $\Delta=\frac{1}{2}\left(\Delta_{1}-\Delta_{2}\right)$ and $\delta=\kappa_{1} \kappa_{2}$. Hence there is instability if $\kappa_{1} \kappa_{2}<0$, and stability if $\kappa_{1} \kappa_{2}>0$. Equations of the form (1.2) have been derived by Mitsudera (1994) and Gottwald and Grimshaw (1999) for certain quasi-geostrophic flows, and by Grimshaw (2000) for a certain threelayer stratified shear flow. The coupled KdV sytem is Hamiltonian, and so conserves "energy"; the system also conserves two Casimirs (the integrals of $A$ and $B$ ) as well as the "momentum" invariant,

$$
P=\int_{-\infty}^{\infty}\left\{\kappa_{1} A_{2}^{2}+\kappa_{2} A_{1}^{2}\right\} d x
$$

Note that $\mathrm{P}$ is sign-definite when the system is linearly stable, but signindefinite if the system is linearly unstable.

Here we shall briefly review the derivation of the coupled KdV system (1.2) for a two-layer quasi-geostrophic flow, and some of the more 
relevant solutions of (1.2). More details can be found in the work of Grimshaw and Gottwald (1999) (hereafter denoted by GG). We shall also describe the relationship between these solutions of the coupled KdV system and the corresponding solutions of the full quasi-geostrophic equations, based on some preliminary numerical studies of the latter system.

\section{TWO-LAYER QUASI-GEOSTROPHIC FLOW}

In order to illustrate the basic concepts in a relatively simple geophysical fluid dynamics context, we introduce the familiar two-layer quasigeostrophic model on a $\beta$-plan, in the Boussinesq approximation when the layer densities are almost equal. We shall use a nondimensional coordinate system, based on a typical horizontal lengthscale $L_{0}$, typical vertical scales for each layer $D_{1}, D_{2}$ with $H_{0}=D_{1}+D_{2}$, and a typical Coriolis parameter $f_{0}$. A typical velocity $U$ is taken to be the maximum of the mean current velocity and the timescale is given by $U / L_{0}$. If we separate the meanflow $U_{1}$ and $U_{2}$ from the perturbation pressure fields $p_{1}$ and $p_{2}$, we obtain the following equations (Pedlosky (1978)) for the nondimensional perturbation pressure fields

$$
\left(\frac{\partial}{\partial t}+U_{n} \frac{\partial}{\partial x}\right) q_{n}+\psi_{n x} Q_{n y}+J\left(\psi_{n}, q_{n}\right)=0,
$$

where $n=1,2$ respectively, and

$$
\begin{array}{r}
q_{n}=\nabla^{2} \psi_{n} \pm F_{n}\left(\psi_{2}-\psi_{1}\right), \\
Q_{n y}=\beta-U_{n y y} \mp F_{n}\left(U_{2}-U_{1}\right) .
\end{array}
$$

with the Jacobian defined by $J(a, b)=a_{x} b_{y}-a_{y} b_{x}$. Here the alternate signs refer to $n=1,2$ respectively. The boundary conditions are $\psi_{1,2}=$ const at $y=-L, 0$. Here the pressure fields are scaled by $\rho_{0} f_{0} U_{0} L_{0}$, where $\rho_{0}$ is a reference density, and, in this quasigeostrophic approximation, also serve as streamfunctions for the velocity fields in each layer. The subscripts 1 and 2 are associated with the upper and lower layers, respectively. We have introduced the nondimensional meridional gradient of planetary vorticity $\beta$, and the Froude-numbers $F_{n}=\left(L_{0} / R_{i}\right)^{2}$, where $R_{i}$ is the internal Rossby radius of deformation for each layer, i.e $R_{i}=f_{0}^{-1} \sqrt{g D_{n}\left(\Delta \rho / \rho_{o}\right)}$, where $\Delta \rho$ is the density difference across the interface.

Our concern here is with weakly nonlinear long waves in a parameter regime where two wave modes have nearly coincident phase speeds. 
Hence we introduce the following scaled variables,

$$
\begin{gathered}
X=\delta x, \quad T=\delta^{3} t, \\
\psi_{n}=\delta^{2} \psi_{n}^{(0)}+\delta^{4} \psi_{n}^{(1)}+\cdots, \\
U_{n}=U_{n}^{(0)}+\delta^{2} U_{n}^{(1)}+\cdots,
\end{gathered}
$$

where $\delta$ is a small parameter, the inverse of which measures the horizontal scale of the waves. This scaling is typical for KdV systems. Next, we rescale the parameters

$$
F_{n} \rightarrow \delta^{2} F_{n}, \quad \beta \rightarrow \delta^{2} \beta .
$$

As we shall see the scaling of the Froude numbers is to ensure that the desired resonance between two waves can be realised. It implies that our model is valid for situations where the internal Rossby radius of deformation is of the order of the long horizontal scale. Further, the scaling of $\beta$ implies that $Q_{n y} \approx-U_{n y y}$ at the lowest order. Substituting this scaling into equation (2.1), we obtain at the lowest order, $\mathcal{O}\left(\delta^{3}\right)$

$$
U_{n}^{(0)} \psi_{n X y y}^{(0)}-U_{n y y}^{(0)} \psi_{n X}^{(0)}=0,
$$

from which we conclude that

$$
\psi_{1}^{(0)}=A(X, T) U_{1}^{(0)}(y) \quad \text { and } \quad \psi_{2}^{(0)}=B(X, T) U_{2}^{(0)}(y) .
$$

Hence the meridional structure of $\psi_{i}$ is entirely determined by the mean currents at the leading order. Note that two independent amplitudes $A(X, T), B(X, T)$ appear, indicating that there is indeed a resonance between two waves, whose phase speeds are identically zero in this present case.

The $\mathcal{O}\left(\delta^{5}\right)$ terms give us two evolution equations for the amplitudes $A, B$ for each layer. We reiterate that the reason for the occurrence of two coupled equations is the scaling of the Froude numbers (2.4), which implies the existence of two independent modes at leading order. We obtain

$$
U_{n}^{(0)} \psi_{n X y y}^{(1)}-U_{n y y}^{(0)} \psi_{n X}^{(1)}+G_{n}=0
$$

where the inhomogeneous terms $G_{n}$ contain the terms $A_{T}, A_{X}, B_{X}$, $A_{X X X}$ and $A_{X}$, or $B_{T}, B_{X}, A_{X}, B_{X X X}$ and $B B_{X}$ respectively, (for details see GG). Applying a compatibility condition to each equation then yields a coupled KdV system, which is just (1.2) on reverting to the unscaled coordinates. Here the coefficients are give by,

$$
I_{n}=\left[U_{n y}^{(0)}\right]_{-L}^{0}, \quad I_{n} \lambda_{n}=\int_{-L}^{0} U_{n}^{(0)^{2}} d y, \quad I_{n} \mu_{n}=-\left[U_{n y}^{(0)^{2}}\right]_{-L}^{0},
$$




$$
\begin{gathered}
I_{n} \Delta_{n}=\int_{-L}^{0}\left(\beta-F_{n} U_{m}^{(0)}\right) U_{n}^{(0)} d y+\left[U_{n}^{(1)} U_{n y}^{(0)}\right]_{-L}^{0}, \\
I_{n} \kappa_{n}=F_{n} \int_{-L}^{0} U_{1}^{(0)} U_{2}^{(0)} d y .
\end{gathered}
$$

Here $n=1,2$ and $m=2,1$. Note that the linear instability criterion $\kappa_{1} \kappa_{2}<0$ is here equivalent to $I_{1} I_{2}<0$ which is just the familiar condition for baroclinic instability in a two-layer system with the present scaling. It should also be noted that in order for the nonlinear coefficients $\mu_{n}$ to be non-zero, the basic flow $U_{n}^{(0)}$ should be assymetric.

\section{INTERACTING SOLITARY WAVES}

The coupled KdV system (1.2) can support a rich variety of solutions. Here we shall describe just one scenario of interest. For more details of this and other dynamics, see GG. In the absence of any coupling between the component equations in (1.2), the system reduces to two KdV equations, each of which can support a solitary wave. In the presence of coupling, we then expect these to interact with each other. This process can be described by seeking asymptotic solutions of the form of solitary wave solutions of the KdV equation, but with an amplitude and speed which are allowed to evolve in time, that is,

$$
\begin{aligned}
& A_{0}\left(B_{0}\right)=a_{n}(t) \operatorname{sech}^{2}\left[w_{n}(t) x-\Phi_{n}(t)\right], \\
& \text { where } \quad a_{n}=12 \frac{\lambda}{\mu} w_{n}^{2} .
\end{aligned}
$$

The time-evolution of the amplitudes $a_{n}(n=1,2)$ and the relative position $\Phi=\Phi_{2}-\Phi_{1}$ are determined by the following set of three ordinary differential equations,

$$
\frac{d a_{n}}{d t}=F_{n}\left(a_{1}, a_{2}, \Phi\right), \quad \frac{d \Phi}{d t}=\Delta_{2}-\Delta_{1}+\frac{\mu_{2}}{3} a_{2}-\frac{\mu_{1}}{3} a_{1} .
$$

where the interaction-integrals are given by

$$
\begin{aligned}
& F_{n}=2 \kappa_{n} a_{m} w_{m} \int_{-\infty}^{\infty} \operatorname{sech}^{2}(\psi) \operatorname{sech}^{2}\left(\psi^{\prime}\right) \tanh \left(\psi^{\prime}\right) d \psi . \\
& \text { where } \quad \psi^{\prime}=\frac{w_{m}}{w_{n}} \psi-w_{m} \Delta \Phi \text { and } \quad n=1,2 ; m=2,1 .
\end{aligned}
$$

The reduced system 3.2 conserves the analogue of the "momentum" $P(1.4)$, namely $\left(\kappa_{2}\left[\lambda_{1}^{2} / \mu_{1}^{2}\right] w_{1}^{3}+\kappa_{1}\left[\lambda_{2}^{2} / \mu_{2}^{2}\right] w_{2}^{3}\right)$, which enables us to reduce the system (3.2) to a planar dynamical system. This resulting planar sytem has been analysed in detail by Gottwald and Grimshaw 

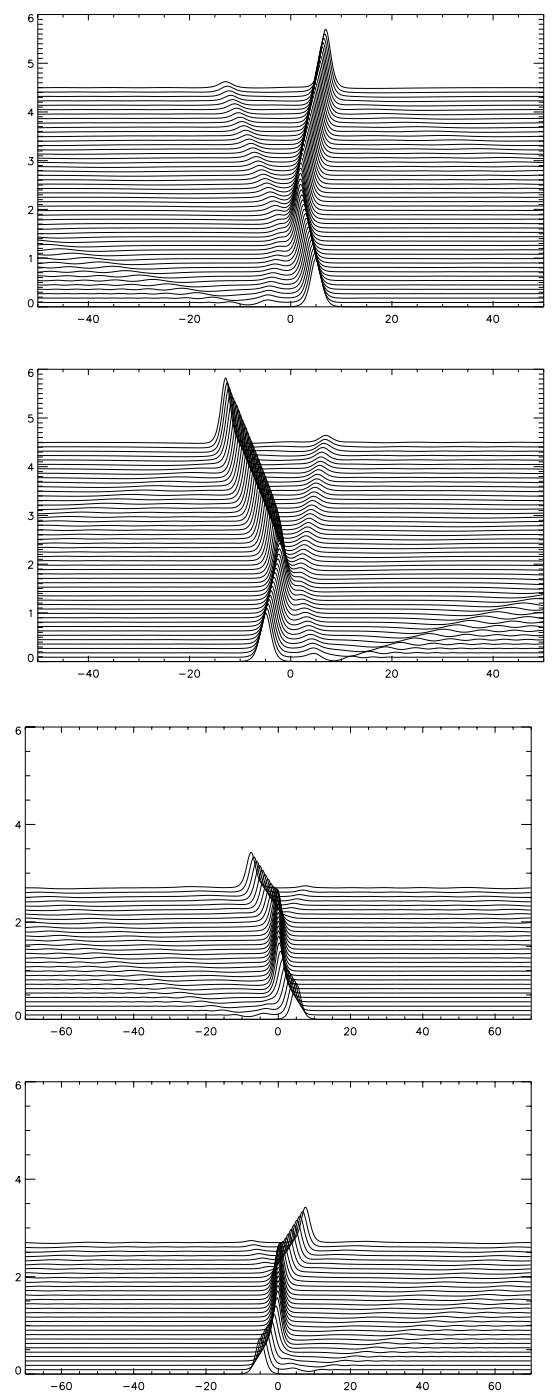

Figure 1 Solitary wave interactions for a saddle point with $\Delta_{1}=-2.25, \Delta_{2}=$ $2.25, \mu_{1}=\lambda_{1}=-1, \mu_{2}=\lambda_{2}=1, \kappa_{1}=-0.2, \kappa_{2}=0.3$. The top two panels show a repulsive regime with initial amplitudes $a_{1}=a_{2}=6.0$ for the upper and lower layer respectively. The lower two panes show a quasi-locked state with initial amplitudes $a_{1}=a_{2}=4.44$. The horizontal axis is the space coordinate $x$ and the vertical axis is the time coordinate $t$. 

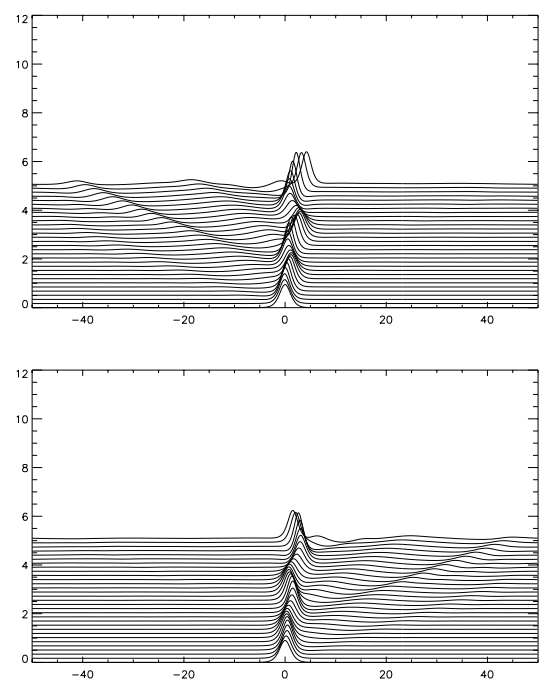

Figure 2 A trapped regime for a stable centre with $\Delta_{1}=-1.7, \Delta_{2}=1.8, \mu_{1}=\lambda_{1}=$ $-1, \mu_{2}=\lambda_{2}=1, \kappa_{1}=0.3, \kappa_{2}=-0.2$, where the amplitudes of the the solitary waves oscillate about $a_{1}=5.7$ and $a_{2}=5.4$. The axes are as in Figure 1, and the upper and lower panels correspond to the upper and lower lavers respectivelv
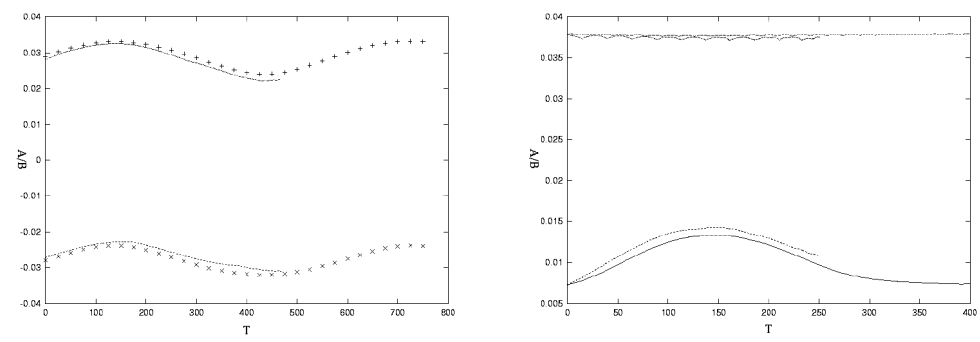

Figure 3 Comparison of the dynamics given by the coupled KdV equations (1.2) and the two-layer quasi-geostrophic system (2.1). The left panel shows a centre, where we plot the amplitude of the solitary waves against the time coordinate; the upper(lower) curves are that for $A(B)$, and the curves which extend for all time are those for the coupled KdV system. The right panel shows a quasi-locked state for a a saddle point configuration.

(1999). Here, we just note that it possesses a critical point $\Delta \Phi=0$ with $w_{1}=w_{2}=w_{0}$ say, which is in fact also an exact solution of the full coupled KdV system (1.2), thus descibing an exact phase-locked solitary wave. A routine stability analysis then shows that this solution is a centre and so stable if $\kappa_{1} \mu_{1} \mu_{2}<0$, but is a saddle-point and so unstable if $\kappa_{1} \mu_{1} \mu_{2}>0$. Typical scenarios describing these two configurations are shown in Figures 1 and 2, these results being obtained by direct numerical simulation of the coupled KdV system (1.2). 
Although the coupled KdV system (1.2) has been systematically derived from the full two-layer quasi-geostrophic system (2.1), there remains a need to verify directly that the scenarios identified in the reduced system can also be found in the full system. To this end we report here some preliminary numerical simulations of the full two-layer quasigeostrophic sytem. Using the results shown in Figures 1,2 as a guide we simulated a stable solitary wave interaction in (2.1) which displays the "centre" scenario, and also an unstable interaction in (2.1) which displays the "saddle-point" interaction. The results are shown in Figure 3.

\section{References}

[1] Baines, P.G. and Mitsudera, H. (1994): On the mechanism of shear flow instabilities, J. Fluid Mech. 276, 327-342.

[2] Craik, A.D.D. (1985): Wave interaction in fluid flows, C.U.P., 332 pp.

[3] Gottwald, G. and Grimshaw, R. (1999): The formation of coherent structures in the context of blocking, J.Atmos Sci., 56, 3663-3678.

[4] Grimshaw, R., (2000): Models for long-wave instability due to a resonance between two waves, in "Trends in Applications of Mathematics to Mechanics", ed. G. Iooss, O. Gues and A. Nouri, Monographs and Surveys in Pure and Applied Mathematics, 106, Chapman 8 Hall/CRC, 183-192.

[5] Helfrich, K.R. and Pedlosky, J. (1993): Time-dependent islated anomolies in zonal stable, inviscid shear flows I. Derivation of the amplitude evolution equations, J Fluid Mech. 251, 377-409.

[6] Hickernell, F., (1983a): The evolution of large-horizontal scale disturbances in marginally stable, inviscid shear flows I. Solutions of the Boussinesq equation, Stud Appl Math., 69, 1-21.

[7] Hickernell, F., (1983b): The evolution of large-horizontal scale disturbances in marginally stable, inviscid shear flows II. Solutions of the Boussinesq equation, Stud Appl Math., 69, 23-39.

[8] Mitsudera, H. (1994): Eady solitary waves: a theory of type B cyclogenesis, J. Atmos. Sci., 57, 734-745.

[9] Pedlosky, J. (1987): Geophysical Fluid Dynamics, 2nd ed. SpringerVerlag, 710pp. 